\title{
The Immobilization of a Transfer Hydrogenation Catalyst on Colloidal Particles
}

\author{
Bas G. P. van Ravensteijn, ${ }^{[a, c]}$ Dirk-Jan Schild, ${ }^{[a]}$ Willem K. Kegel, ${ }^{*[a]}$ and \\ Robertus J. M. Klein Gebbink ${ }^{*[b]}$
}

\begin{abstract}
In this paper, we report a new synthetic procedure to immobilize a transfer hydrogenation catalyst on the surface of colloidal polystyrene particles. Using supports of colloidal dimensions allows for combining a relatively high surface area for catalyst binding, mobility of the catalyst, and facile recovery by centrifugation or sedimentation. The immobilization procedure relies on the covalent attachment of terpyridine moieties on the particle surface. These immobilized terpyridines are subsequently employed as colloidal ligands, which participate in the formation of an active ruthenium-based transfer hydrogenation catalyst. The resulting functional colloidal particles successfully catalyze the transfer hydrogenation of acetophenone
\end{abstract}

with 2-propanol as the hydrogen donor. Thorough analysis of the chemical composition of the colloidal surface led to the determination of the catalyst loading per particle. This enabled us to conduct reference hydrogenations with equal concentrations of the homogeneous transfer hydrogenation catalyst to probe the effect of the immobilization procedure on the catalytic activity. Despite a decrease in transfer hydrogenation activity, full acetophenone conversion is still achievable within $24 \mathrm{~h}$. Preliminary experiments show that the catalytic colloids are recyclable without significant loss of transfer hydrogenation activity.

\section{Introduction}

Colloids refer to particles with linear dimensions between 10 and $1000 \mathrm{~nm}$, which are typically dispersed into a continuous medium (colloidal dispersion). ${ }^{[1]}$ Therefore, the colloidal size regime exists in between nanoscale and macroscopic objects, enabling colloidal particles to combine the properties of these two extreme length scales in a unique fashion. Similarly to nanoparticles, colloids are small enough to be subjected to Brownian motion, causing persistent movement of the particles. Gravitational forces acting on the objects are small, keeping them well-dispersed in the continuous medium. In contrast to nanoparticles, the relatively large dimensions of colloids facilitate separation from the continuous medium by simple cen-

[a] Dr. B. G. P. van Ravensteijn, D.-J. Schild, Prof. Dr. W. K. Kegel Van't Hoff Laboratory for Physical and Colloid Chemistry

Debye Institute for NanoMaterials Science Utrecht University

Padualaan 8, 3584 CH, Utrecht (The Netherlands) E-mail:w.k.kegel@uu.nl

[b] Prof. Dr. R. J. M. Klein Gebbink Organic Chemistry and Catalysis

Debye Institute for NanoMaterials Science Utrecht University

Universiteitsweg 99, 3584 CG, Utrecht (The Netherlands) E-mail:r.j.m.kleingebbink@uu.nl

[c] Dr. B. G. P. van Ravensteijn

Current address: Department of Chemical Engineering University of California Santa Barbara Santa Barbara, CA 93105 (USA)

$\square$ Supporting information for this article can be found under http:// dx.doi.org/10.1002/cctc.201601096. It includes the calibration curves of the $\mathrm{Fe}^{2+}$ titration experiment used to determine the terpyridine surface density and presumable catalytic mechanism for the employed catalyst. trifugation or filtration. Although particles of colloidal dimensions have less surface area per gram of material compared to nanoparticles, the available surface is significantly higher than for macroscopic objects.

Because of these distinctive properties of colloidal systems, they are applied in a wide variety of fields, ranging from fundamental model systems for atoms/molecules to food applications and optics. ${ }^{[2,3]}$ This broad applicability is further facilitated by the fact that colloidal particles can be extensively tuned in terms of their shape, bulk material, and chemical surface properties. In particular, the introduction of functional chemical handles on the surface of colloidal particles makes them valuable platforms for solid-phase reactions, ${ }^{[4]}$ bioassays, ${ }^{[5]}$ and drug delivery. ${ }^{[6]}$

The intermediate size of colloidal objects combined with chemical surface reactivity make colloids in principle also a promising target for catalyst immobilization. ${ }^{[7]}$ In general, immobilization of catalysts onto solid supports provides a route toward hybrid catalytic systems to simultaneously combine the advantages and circumvent the disadvantages associated with purely homogeneous and heterogeneous catalysts. By tethering homogeneous complexes to a solid support, a hybrid catalytic system is obtained in which activity and selectivity are governed by the well-defined structure of the immobilized molecular species, while recycling is promoted by their attachment to a (solid) carrier. ${ }^{[8]}$

For the commonly applied immobilization platforms, such as nanoparticles, dendrimers, and macroscopic surfaces, a tradeoff between high surface area/catalyst mobility and easy catalyst recovery is unavoidable. In contrast, the intermediate size 
range of colloidal objects circumvents this trade-off. High surface areas for catalyst binding, catalyst mobility owing to Brownian motion of the supporting particles, and facile recovery from the reaction mixture by centrifugation or filtration are all combined in one carrier platform. Colloidal particles can therefore be regarded as the ultimate compromise between macroscopic surfaces, nanoparticles, and dendritic structures as catalyst supports.

To explore and exploit the benefits of colloids as catalyst carriers, we immobilized a transfer hydrogenation catalyst on the surface of colloidal polystyrene particles. Transfer hydrogenations are synthetically attractive reactions in which unsaturated compounds, for example, ketones, are reduced by employing secondary alcohols as hydrogen donors. ${ }^{[9]}$ Conducting hydrogenation reactions in this fashion has the clear advantage that the use of highly flammable and explosive hydrogen gas or hazardous reducing agents is prevented. A large number of organometallic complexes that catalyze (enantioselective) transfer hydrogenation are currently reported in the literature. ${ }^{[9]}$ For this study, we selected a ruthenium-based catalyst reported by Kelson et al., which comprises two pyridonate moieties next to a terpyridine ligand (Scheme 1). ${ }^{[10]}$ Although the selected catalyst is not among the most active transfer hydrogenation catalysts reported to date, ${ }^{[9]}$ the facile synthesis of this ruthenium complex and no need for in situ preparation of the active catalytic species, allows us to neatly illustrate our novel immobilization strategy.

To realize immobilization of the transfer hydrogenation catalyst, terpyridine moieties were covalently tethered to the surface of colloidal polystyrene particles. These immobilized terpyridines were subsequently exploited as colloidal ligands for the formation of the complete transfer hydrogenation catalyst in an analogous synthesis procedure as described for the homogeneous counterpart. ${ }^{[10]}$

To investigate the catalytic activity of the supported catalyst and to probe the effect of the immobilization, the transfer hydrogenation of acetophenone to 1-phenylethanol with 2-propanol as the hydrogen donor was used as model reaction. We showed that upon immobilization, catalytic activity was preserved. Separation of the catalytic colloids by simple centrifugation and reusing them in a subsequent transfer hydrogenation showed the potential of recycling the immobilized catalysts. These preliminary results provide proof of principle for our novel immobilization strategy.

\section{Results and Discussion}

\section{Synthesis of homogeneous transfer hydrogenation catalyst (TPR) and immobilization strategy}

A ruthenium-based transfer hydrogenation catalyst (trans-[(terpy) Ru( $\left.\left(\mathrm{NC}_{5} \mathrm{H}_{4} \mathrm{O}-\kappa \mathrm{N}\right)_{2}\left(\mathrm{OH}_{2}\right)\right]$, TPR) previously reported by Kelson et al. ${ }^{[10]}$ was selected to investigate the feasibility of our immobilization procedure. The active complex was easily obtained by a two-step synthesis process. First, $\mathrm{RuCl}_{3}$ was coordinated to $2,2^{\prime}: 6^{\prime}, 2^{\prime \prime}$-terpyridine (terpy). The coordinated ruthenium center was further decorated by two auxiliary pyridonate ligands upon addition of 2-hydroxypyridine and base. This second step completed the formation of the TPR complex, which was readily isolated by filtration in the form of darkpurple crystals. Neither reaction step required demanding reaction conditions, for example, complete exclusion of water or oxygen, and the final complex was stable under ambient atmosphere. These features ensure that the synthesis procedure

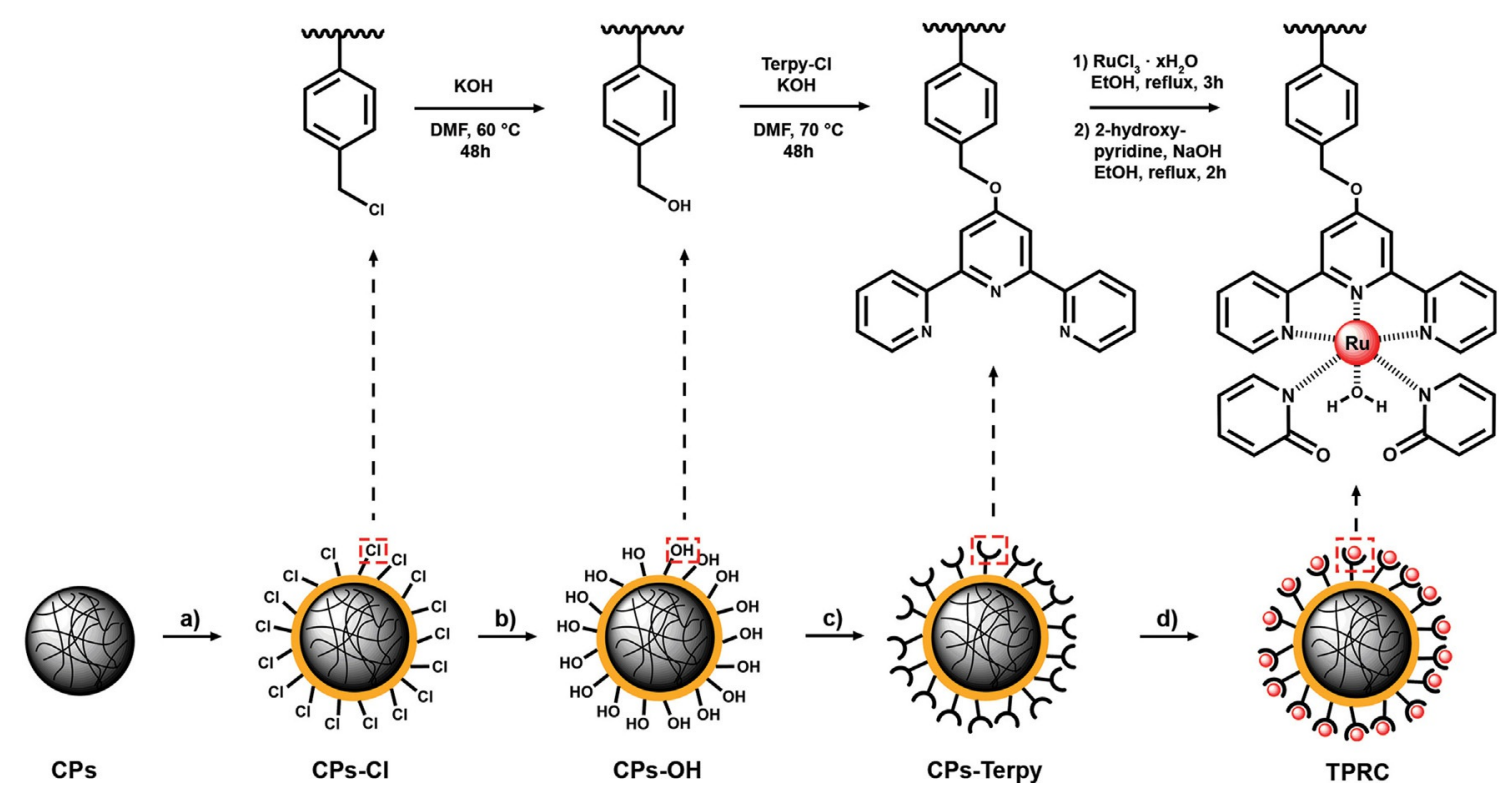

Scheme 1. Synthetic strategy to immobilize the transfer hydrogenation catalyst of choice on the surface of colloidal polystyrene particles. Step (a): seededemulsion polymerization of 4-vinylbenzyl chloride (VBC) and divinylbenzene (DVB) in the presence of cross-linked polystyrene particles (CPs). Step (b): hydrolysis of the surface chlorine groups by reaction with $\mathrm{KOH}$ in dimethylformamide (DMF). Step (c): attachment of $4^{\prime}$-chloro-2,2':6',2'-terpyridine (Terpy-Cl) to the hydrolyzed particle surface by reaction in DMF in the presence of $\mathrm{KOH}$. Step (d): coordination of $\mathrm{RuCl}_{3}$ to the immobilized terpyridine ligands followed by coordination of two auxiliary pyridonate ligands after addition of 2-hydroxypyridine and an aqueous $\mathrm{NaOH}$ solution. 
is easily adaptable towards an immobilized analog of TPR. Employing polystyrene particles functionalized with covalently bound terpyridine moieties is a key step in the immobilization strategy. We hypothesize that the surface-tethered terpyridines could subsequently be exploited as colloidal ligands in the formation of the active transfer hydrogenation catalyst in a similar fashion as described above for the homogeneous counterpart.

\section{Synthesis of terpyridine-functionalized polystyrene colloids (CPs-Terpy)}

Terpyridine-functionalized colloids were successfully synthesized in four steps, which are schematically depicted in Scheme 1. The first step comprised a straightforward emulsion polymerization of styrene and divinylbenzene (DVB) to yield monodisperse, cross-linked seed particles (CPs) with a diameter of approximately $350 \mathrm{~nm} .^{[11]}$

Benzyl chloride moieties were introduced on the surface of these polystyrene particles by conducting a seeded-emulsion polymerization with 4-vinylbenzyl chloride (VBC) and DVB as monomers (Scheme 1, step a). ${ }^{[11]}$ Proof of successful incorporation of the chlorine functionality was provided by both infrared (IR) spectroscopy (Figure 1, blue spectrum) and X-ray photoelectron spectroscopy (XPS; Figure 2, red spectrum). Comparing the IR spectra of the bare polystyrene seeds (Figure 1, red spectrum) and the chlorinated particles $(\mathrm{CPs}-\mathrm{Cl})$ revealed the appearance of a distinct new signal at $1266 \mathrm{~cm}^{-1}$, which corresponds to the $-\mathrm{CH}_{2}-\mathrm{Cl}$ vibration of the incorporated vinylbenzyl chloride. Binding energies of 200 and $270 \mathrm{eV}$ in the XPS spectrum corresponding to chlorine were detected, providing additional evidence that these chlorinated moieties were present at the particle surface, as XPS is a surface elemental analysis technique with a penetration depth of only $1-10 \mathrm{~nm}$. The detected carbon signal $(284 \mathrm{eV})$ originated from the polystyrene core of the colloids. Oxygen was detected owing to the presence of sulfate and sulfonate groups at the surface of the colloids. These moieties originate from the initiator system (KPS and $\mathrm{NaHSO}_{3}$ ) employed in the emulsion polymerizations

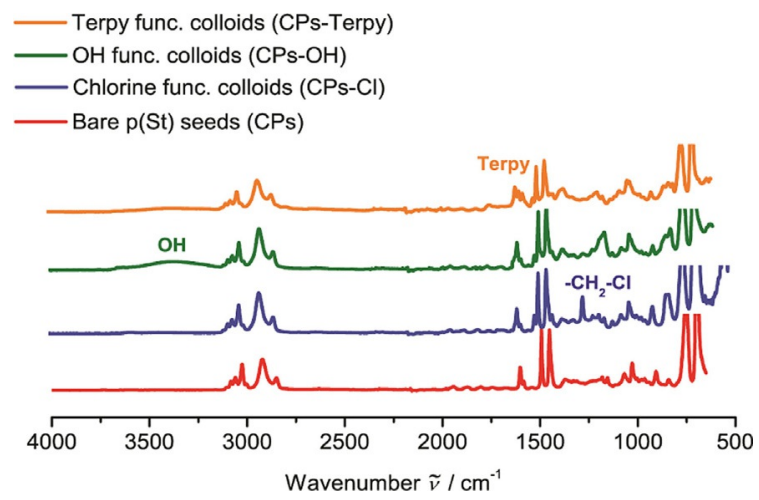

Figure 1. Infrared (IR) spectra of the bare polystyrene seeds (CPs, red), chlorinated core-shell particles ( $\mathrm{CPs}-\mathrm{Cl}$, blue), the particles obtained after hydrolysis of the surface chlorine groups (CPs-OH, green), and the terpyridine-functionalized polystyrene colloids (CPs-Terpy, orange). Characteristic signals are labelled with the corresponding chemical entity causing the vibration.

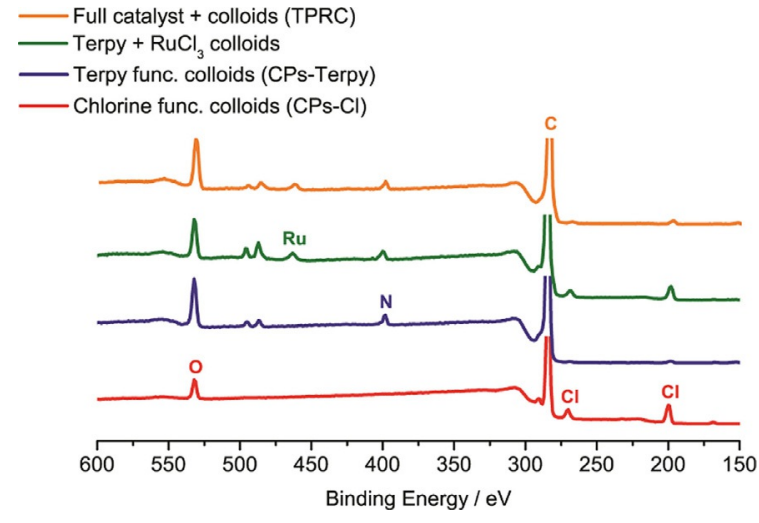

Figure 2. X-ray photoelectron spectroscopy (XPS) spectra of chlorinated core-shell particles (CPs-Cl, red), terpyridine-functionalized polystyrene colloids (CPs-Terpy, blue), terpyridine-containing colloids after the complexation reaction with $\mathrm{RuCl}_{3}$ (green), and the spectrum obtained from particles equipped with the complete transfer hydrogenation catalyst on their surface (TPRC, orange). Characteristic signals are labelled with the corresponding element appearing at that binding energy.

to form the bulk and the outer chlorinated shell of the chlorinated particles (see the Experimental Section). ${ }^{[12]}$

The introduction of chlorine groups provides a chemical handle for further chemical modification. As the chlorine moieties are localized on the surface of the prepared particles, all subsequent chemistry is strictly confined to the surface as well. This is a major advantage, as it will ensure good catalyst accessibility after completing the immobilization strategy (Scheme 1) and therefore optimal catalytic activity of the colloidal particles.

The surface chlorine groups of $\mathrm{CPs}-\mathrm{Cl}$ were subsequently hydrolyzed by addition of $\mathrm{KOH}$ (Scheme 1, step b), yielding hydroxylated polystyrene particles ( $\mathrm{CPs}-\mathrm{OH})$. After the reaction, IR spectroscopy revealed the disappearance of the characteristic $-\mathrm{CH}_{2}-\mathrm{Cl}\left(1266 \mathrm{~cm}^{-1}\right)$ vibration and the appearance of a broad $\mathrm{OH}$ signal at $3400 \mathrm{~cm}^{-1}$ (Figure 1, green spectrum), indicating successful hydrolysis.

In the final step required for the preparation of terpyridinefunctionalized colloids, the surface hydroxyl groups were exploited to covalently attach $4^{\prime}$-chloro- $2,2^{\prime}: 6^{\prime}, 2^{\prime \prime}$-terpyridine through a nucleophilic aromatic substitution reaction (Scheme 1, step c). The presence of the terpyridine moieties on the surface could again be confirmed with IR spectroscopy (Figure 2, orange spectrum). After terpyridine coupling, the IR spectrum showed a significant lowering of the $\mathrm{OH}$ signal intensity and the appearance of a signal at $1562 \mathrm{~cm}^{-1}$, characteristic for the $\mathrm{C}=\mathrm{N}$ vibrations of terpyridines. XPS analysis provided additional proof for the successful attachment of terpyridine. A sharp decrease of the chlorine signals (200 and $270 \mathrm{eV}$ ) was accompanied by the appearance of a nitrogen signal at $398 \mathrm{eV}$ (Figure 2, blue spectrum). The signals around $490 \mathrm{eV}$ represent Sn detector impurities.

Final confirmation of the successful immobilization of the terpyridine ligands was provided by the addition of $\mathrm{FeCl}_{2}$ to a dispersion of presumably terpyridine-functionalized particles. Before addition, the dispersion was white in appearance (Fig- 

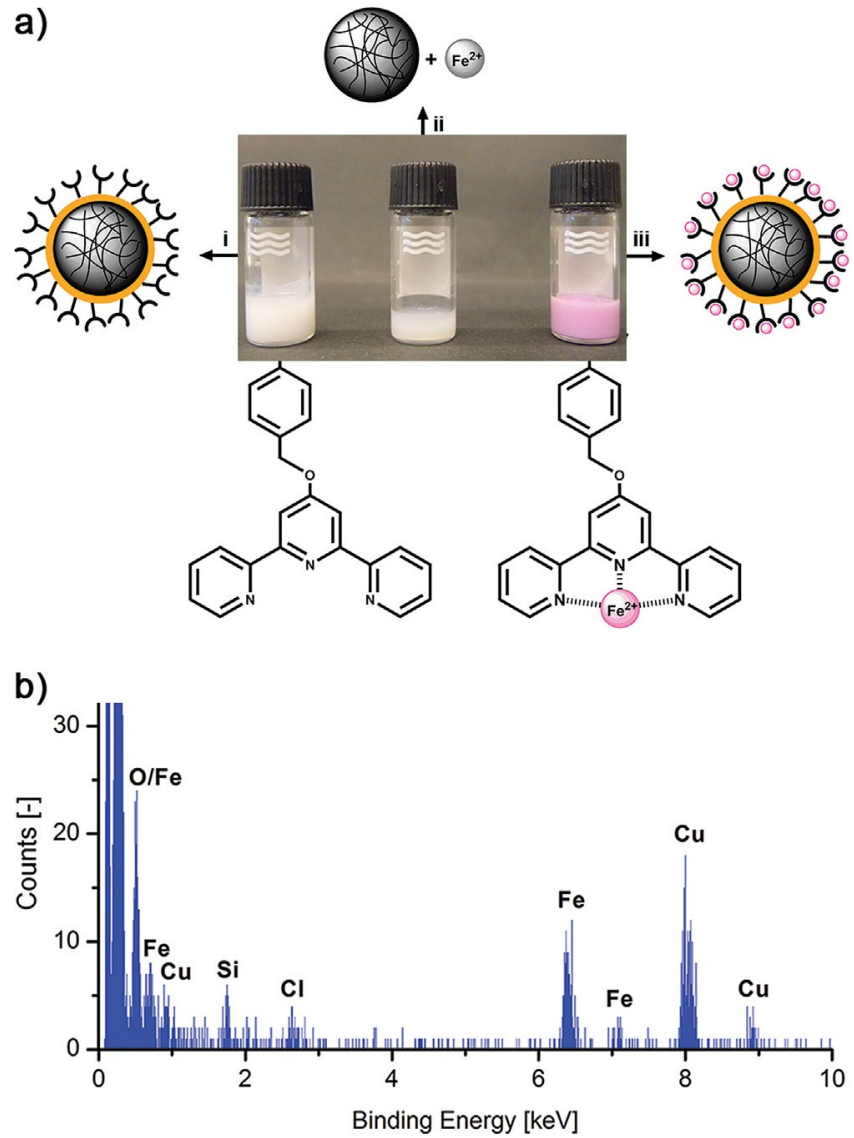

Figure 3. (a) Macroscopic appearance of terpyridine-functionalized particles (CPs-Terpy) without the addition of $\mathrm{FeCl}_{2}$ (left, vial i), non-functionalized particles (CPs) in the presence of $\mathrm{FeCl}_{2}$ (middle, vial ii), and the pink dispersion obtained after adding $\mathrm{FeCl}_{2}$ to a CPs-Terpy dispersion (right, vial iii). The pink color is characteristic for $\mathrm{Fe}^{2+}$-terpyridine complexes. (b) Energy dispersive $\mathrm{X}$-ray (EDX) spectrum of the iron-loaded terpyridine-functionalized polystyrene particles.

ure $3 \mathrm{a}$, vial i). Upon treating this white dispersion with $\mathrm{FeCl}_{2}$, an intense pink color emerged (Figure $3 \mathrm{a}$, vial iii), which is characteristic for terpyridine-Fe ${ }^{2+}$ complexes. ${ }^{[13]}$ Centrifugation of the colored dispersion yielded an intensely pink sediment and a colorless supernatant, indicating that the terpyridine$\mathrm{Fe}^{2+}$ complexes were indeed attached to the colloids. As a reference, $\mathrm{FeCl}_{2}$ was added to a dispersion containing non-functionalized (CPs) particles. As anticipated, no color change was observed in this situation (Figure $3 \mathrm{a}$, vial ii).

The complexation capability of the surface-tethered terpyridine groups was further confirmed by using energy dispersive $X$-ray spectroscopy (EDX). The particles treated with $\mathrm{FeCl}_{2}$ were measured by using this elemental analysis technique, resulting in a spectrum clearly revealing iron related signals located at 6.4 and $7.1 \mathrm{keV}$ (Figure $3 \mathrm{~b}$ ). The copper and silicon signals originated from the grid on which the sample was placed and detector impurities, respectively.

With experimental evidence for successful terpyridine immobilization and its capability to bind metal ions at hand, we proceeded by estimating the actual terpyridine surface density on CPs-Terpy. Knowledge about the ligand density is useful for calculating the quantities of reactants required for the subsequent reaction steps to complete catalyst formation. Furthermore, the terpyridine density sets an upper limit for the catalyst loading per colloidal particle. Having this estimation for the catalyst loading allows for fair comparison between the catalytic activity of the homogeneous and immobilized catalyst, as transfer hydrogenation reactions with equal concentrations of molecular catalytic species can be conducted.

To this end, a titration experiment was designed in which $\mathrm{FeCl}_{2}$ was added to a CPs-Terpy dispersion (Scheme 2) in a stepwise fashion. After each addition step, the dispersion was stirred to allow for the iron ions to coordinate to vacant terpyridines. After complex formation, the dispersions were centrifuged and the supernatants were tested for residual iron ions by means of UV/Vis spectroscopy (see the Supporting Information for details). Detection of $\mathrm{Fe}^{2+}$ ions was possible after addition of 1,10-phenanthroline. Free $\mathrm{Fe}^{2+}$ ions coordinate to 1,10phenanthroline, generating a highly UV-active complex, allowing for the detection of iron ions down to ppm levels. ${ }^{[14]}$ This procedure was repeated until a certain concentration of free

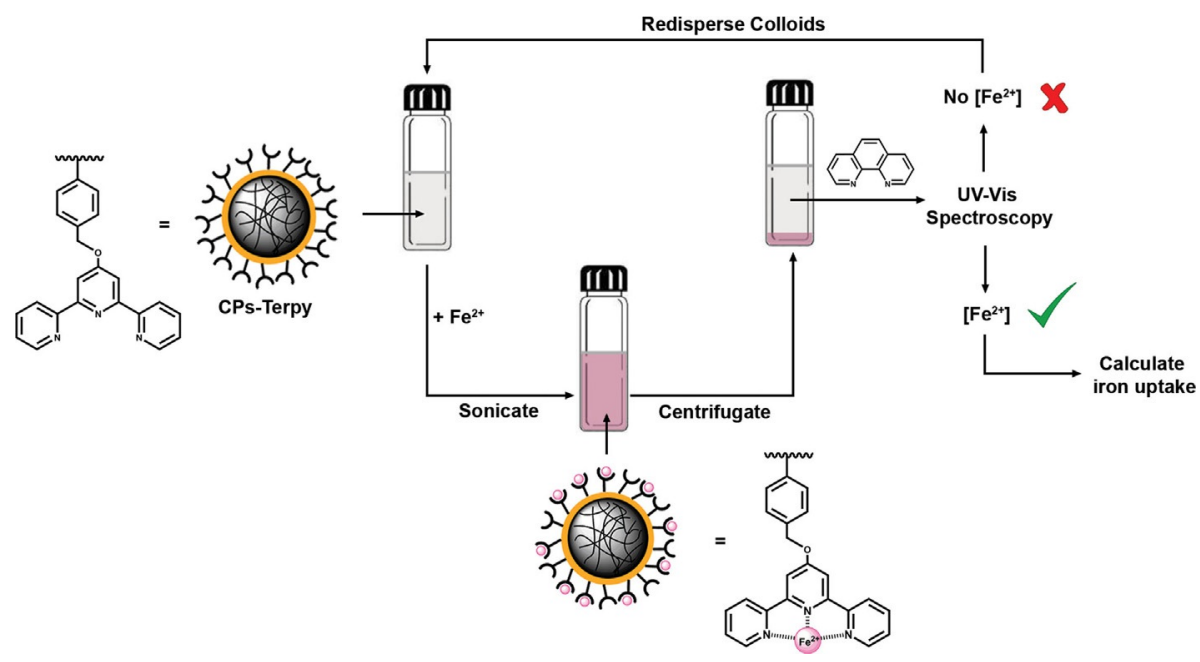

Scheme 2. Schematic representation of the performed iron $\left(\mathrm{Fe}^{2+}\right)$ titration experiment to determine the ligand surface density on terpyridine-functionalized polystyrene particles (CPs-Terpy). 
iron ions was measured. Based on the total amount of ions added to the particles minus the free ions which were measured in the last titration step, the total iron uptake per particle could be determined (see Experimental Section for details on this calculation). This calculation yielded a surface density of one terpyridine moiety per $4 \mathrm{~nm}^{2}$. Although this density is probably a slight overestimation owing to, for example, nonspecific ion adsorption, it indicated the colloidal surface was densely packed with terpyridines.

\section{Exploiting CPs-Terpy as colloidal ligands for the preparation} of immobilized transfer hydrogenation catalysts (TPRC)

With the successful introduction of terpyridine ligands to the surface of our polystyrene colloids and an estimation of their surface density, the final reaction steps to complete the immobilization strategy were performed (Scheme 1). Analogous to the synthesis of the homogenous complex, the first step was coordination of $\mathrm{RuCl}_{3}$ to the surface-bound terpyridines (Scheme 1, step d). Indications for successful coordination of ruthenium were provided by XPS measurements. The spectrum obtained after mixing CPs-Terpy particles with $\mathrm{RuCl}_{3}$ (Figure 2, green spectrum) showed both the appearance of a ruthenium signal $(461 \mathrm{eV})$ and an increase in the chlorine content caused by the auxiliary chlorines coordinated to the ruthenium center. Addition of 2-hydroxpyridine and $\mathrm{NaOH}$ to complete the formation of the catalyst, resulted in a decrease of the chlorine content as observed with XPS (Figure 2, orange spectrum). This is in agreement with replacement of the chlorines by two pyridonate ligands (Scheme 1). The nitrogen and ruthenium signals remained present, suggesting complete catalyst formation.

Although the XPS results provided compositional information, no structural details could be extracted from this data. However, the dispersion obtained after the complete catalyst immobilization procedure displayed a purple color, resembling the color of the molecular catalyst. The match in color is a strong indication that ruthenium is present in the expected oxidation state +2 and that the measured elemental composition indeed corresponds to the formation of the active catalytic complex. Indisputable evidence for the immobilization of the desired complex will be provided by measuring the transfer hydrogenation activity of the synthesized colloidal system.

From a molecular and chemical compositional point of view, catalyst immobilization appeared successful. However, to exploit the colloids as an immobilization platform, the particles themselves should still be intact after the chemical treatments required for catalyst synthesis. To verify the robustness of the particles, transmission electron microscopy (TEM) was used to image the colloids throughout the complete immobilization procedure (Figure 4).

The bare polystyrene particles (CPs, Figure $4 \mathrm{a}$ ), as well as the chlorinated particles (CPs-Cl, Figure $4 \mathrm{~b}$ ) were smooth and spherical in appearance. After hydrolysis of the surface chlorine groups (Figure $4 \mathrm{c}$ ) and introduction of the terpyridine ligands (Figure $4 \mathrm{~d}$ ), the colloidal surface became significantly rougher. As both the hydrolysis and terpyridine coupling were performed in DMF, this surface texture was probably caused by the dissolution of non-cross-linked polymer present in the interior of the polystyrene colloids into the continuous phase (DMF is capable of dissolving linear polystyrene). Coordination of the ruthenium center and the auxiliary pyridonate ligands did not led to a significant change in particle morphology and rough polystyrene colloids were obtained as final product (Figure $4 \mathrm{e}$ ). Capturing an image of the colloids in scanning mode (STEM) underlines the presence of a textured surface even more (Figure $4 \mathrm{f}$ ).

From this series of TEM images, we concluded that the bulk of the polystyrene carrier colloids survived all reactions required for catalyst immobilization. Regardless of the partial dis-
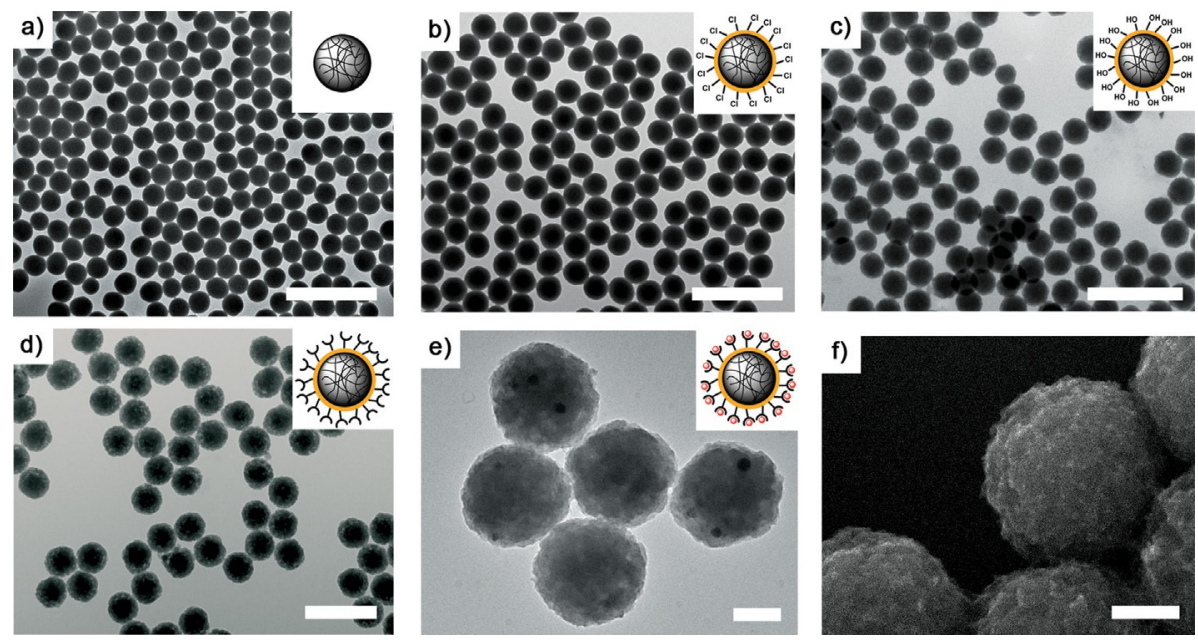

Figure 4. Transmission electron microscopy (TEM) images of colloidal particles throughout the catalyst immobilization procedure. For panels a-e, a schematic representation of the particles (see Scheme 1) is added in the top right corner. (a) Bare cross-linked polystyrene colloids (CPs). (b) Chlorinated polystyrene particles (CPs-Cl). (c) Particles obtained after hydrolysis of the surface benzyl chlorine groups (CPs-OH). (d) Colloids functionalized with immobilized terpyridine ligands (CPs-Terpy). (e) Colloidal particles equipped with the full transfer hydrogenation catalyst on their surface (TPRC). (f) Scanning TEM (STEM) image of TPRC colloids. The scanning mode underlines the surface roughness of the carrier particles. Scale bars in panels $a, b, a n d ~ c=1 \mu m$, panel $d=0.5 \mu m$, and panels e and $\mathrm{f}=0.1 \mu \mathrm{m}$. 
solution of linear polystyrene polymers and the resulting development of surface roughness, the catalytic moieties are still tethered to a relatively heavy carrier. Easy separation of the catalyst from the reaction mixture by centrifugation is therefore anticipated. Furthermore, the developed surface roughness might even be beneficial as it significantly enlarges the available surface area and therefore the potential catalyst loading per particle.

\section{Transfer hydrogenation with TPR}

To probe the influence of the immobilization procedure on the catalytic activity, transfer hydrogenations catalyzed by the homogeneous TPR complex served as a natural reference point. Acetophenone was selected as substrate, as it readily reacts in transfer hydrogenations and is commonly used as a model hydrogen acceptor. ${ }^{[9 f, 15]}$

The catalytic transfer hydrogenations conducted with TPR are summarized in Table 1 . To verify the catalytic activity of the

\begin{tabular}{|c|c|c|c|c|c|c|}
\hline & & & & $\begin{array}{c}\text { Cat. } \\
\text { Base } \\
\end{array}$ & $\stackrel{\mathrm{OH}}{\mathrm{O}}$ & \\
\hline Entry & $\begin{array}{l}\text { TPR } \\
{[\mu \mathrm{mol}]}\end{array}$ & $\begin{array}{l}\mathrm{NaOH} \\
{[\mu \mathrm{mol}]}\end{array}$ & $\begin{array}{l}\text { Aceto- } \\
\text { phenone } \\
{[\mu \mathrm{mol}]}\end{array}$ & $\begin{array}{l}\mathrm{TPR} / \mathrm{NaOH} / \\
\text { ketone ratio }\end{array}$ & $\begin{array}{l}\text { Conversion } \\
\text { after } 2 \mathrm{~h} \\
{[\%]}\end{array}$ & $\begin{array}{l}\text { Conversion } \\
\text { after } 24 \mathrm{~h} \\
{[\%]}\end{array}$ \\
\hline 1 & $10^{[\mathrm{b}]}$ & 200 & 2100 & $1: 20: 210$ & 100 & n.d. \\
\hline 2 & $10^{[b, c]}$ & 200 & 2100 & $1: 20: 210$ & 19 & n.d. \\
\hline 3 & 0.2 & 0 & 40 & $1: 0: 200$ & 0 & 0 \\
\hline 4 & 0.2 & 4 & 40 & $1: 20: 200$ & 7 & 46 \\
\hline 5 & 0.2 & 100 & 40 & $1: 500: 200$ & 27 & 100 \\
\hline 6 & 0.2 & 200 & 40 & 1:1000:200 & 62 & $100^{[\mathrm{d}]}$ \\
\hline
\end{tabular}

[a] All reactions were conducted in 2-propanol; total reaction volume was $5 \mathrm{~mL}$. [b] Degassed as solid. [c] No measures were taken to exclude oxygen from the reaction mixture. [d] Full conversion was reached before measuring. Conversions were determined with gas chromatography-mass spectrometry (GC-MS). n.d. = not determined.

complex, we reproduced the hydrogenation of acetophenone reported by Kelson et al. (Table 1, entry 1). ${ }^{[10]}$ In agreement with the reported results, full conversion was reached within $2 \mathrm{~h}$ by using a catalyst concentration of $2 \mathrm{~mm}$, showing the transfer hydrogenation capability of the prepared complex. However, to be able to employ TPR in its immobilized form, significant reaction rates should be maintained upon drastically lowering the catalyst concentration. The high catalyst loadings used by Kelson et al. are unfeasible for the immobilized variant, as both the number of TPR complexes per particles and the number of colloids in the reaction mixture are limited. The limited surface area of the polystyrene particles restricts the maximal catalyst loading per particle, whereas the overall solid content of the dispersion should be kept low enough to prevent high viscosity and related mixing problems. Therefore, we chose to lower the concentration of TPR by a factor of 50 to a catalyst concentration equal to $0.04 \mathrm{~mm}$. Colloidal dispersions with an equivalent number of catalytic complexes can be prepared without any solid content related problems. Unsurprisingly, a decrease in TPR concentration translated into lower hydrogenation rates. Compared with the full conversion measured after $2 \mathrm{~h}$ for the higher catalyst loading (Table 1, entry 1), only $46 \%$ of the acetophenone was converted after $24 \mathrm{~h}$ when $0.04 \mathrm{~mm}$ TPR was used (Table 1, entry 4).

According to Baratta et al., we should be able to (partially) compensate for the decrease in catalytic activity upon lowering the catalyst concentration by increasing the concentration of base in the reaction mixture. ${ }^{[16]}$ They reported that these transfer hydrogenations typically follow second-order kinetics in which the reaction rate depends on both the catalyst and the base concentration. ${ }^{[16]}$ In agreement with the findings of Baratta et al., significantly higher conversions were measured upon raising the concentration of $\mathrm{NaOH}$ and full conversion could be reached within $24 \mathrm{~h}$ even at these low catalyst loadings. The key role of base was demonstrated by the complete lack of product formation if the transfer hydrogenation reactions were attempted in the absence of base (Table 1, entry 3 ). Clearly, the strong dependence of the catalytic activity on the base concentration allows for performing transfer hydrogenation reactions with reasonable rates at low catalyst loadings. This feature is beneficial for the hydrogenations catalyzed by the immobilized variant of TPR, where the restricted number of catalytic complexes can be easily counteracted by increasing the base concentration.

\section{Transfer hydrogenation with TPRC}

With the transfer hydrogenations performed with TPR as a reference point for the maximal achievable catalytic activity, we proceeded to the TPRC catalyzed reactions. These hydrogenations were performed under the same conditions as applied for the experiments with TPR and are summarized in Table 2. For all transfer hydrogenations, a maximal catalyst loading based on the estimated surface density of terpyridine was assumed. This translates to one catalytic species per $4 \mathrm{~nm}^{2}$. As the reactions were conducted in dispersions with a solid con-

Table 2. Summary of transfer hydrogenations conducted with acetophenone as substrate, 2-propanol as hydrogen donor, and the immobilized catalyst (TPRC). ${ }^{\left[{ }^{[a]}\right.}$

\begin{tabular}{llcllcc} 
Entry & TPR & $\mathrm{NaOH}$ & $\begin{array}{l}\text { Aceto- } \\
\text { phenone } \\
{[\mu \mathrm{mol}]}\end{array}$ & $\begin{array}{l}\mathrm{TPR} / \mathrm{NaOH} / \\
\text { ketone ratio }\end{array}$ & $\begin{array}{l}\text { Conversion } \\
\text { after } 2 \mathrm{~h} \\
{[\%]}\end{array}$ & $\begin{array}{l}\text { Conversion } \\
\text { after 24 } \mathrm{h} \\
{[\%]}\end{array}$ \\
\hline 1 & 0.2 & 0 & 40 & $1: 0: 200$ & 0 & 0 \\
2 & 0.2 & 4 & 40 & $1: 20: 200$ & n.d. & 21 \\
3 & 0.2 & 100 & 40 & $1: 500: 200$ & 5 & 40 \\
4 & 0.2 & 200 & 40 & $1: 1000: 200$ & 18 & 100 \\
5 & $0.2^{[b]}$ & 200 & 40 & $1: 1000: 200$ & n.d. & 83
\end{tabular}

[a] All reactions were conducted in 2-propanol; total reaction volume was $5 \mathrm{~mL}$. Reaction mixtures contained $0.4 \mathrm{wt} \%$ of TPRC colloids. [b] Immobilized catalyst was reused from the reaction performed in entry 4 . Conversions were determined with gas chromatography-mass spectrometry (GCMS). n.d. = not determined. 
tent of $0.4 \mathrm{wt} \%$, the resulting reaction mixtures contained an equivalent of $0.04 \mathrm{~mm}$ immobilized TRP complexes.

Analogous to the catalytic reactions with the homogeneous complex (Table 1, entry 3 ), the addition of base was crucial to obtain any hydrogenation activity (Table 2, entry 1). Entries 1-4 in Table 2 clearly demonstrate that the catalytic action was preserved after immobilization of the TPR complex. However, the activity decreased compared with the homogeneous counterpart (Table 1, entries 3-6) as was deduced from the measured conversions after 2 and $24 \mathrm{~h}$.

The true reason for the decrease in catalytic activity remains speculative and is difficult to assess. One of the most significant factors might be the change in electronic environment caused by the charged polystyrene surface on which the catalysts are immobilized. These negative surface charges originate from the initiator system employed in the seeded-emulsion polymerization to coat the particles with a chlorinated layer (see the Experimental Section). The initiating system, consisting of KPS and $\mathrm{NaHSO}_{3}$, decomposes to generate sulfate and bisulfite radicals, capable of starting the styrene polymerization. This yields polymers with charged sulfate or sulfonate moieties, which will preferentially reside at the surface instead of in the highly hydrophobic interior of the polystyrene particles. The presence of these surface charges, although key for ensuring colloidal stability and preventing aggregation of the carrier particles, may result in lowering of the local hydroxyl concentration in close proximity to the colloidal surface owing to electrostatic repulsion. ${ }^{[17]}$ As the transfer hydrogenation rate is highly dependent on the base concentration (Table 1, entries 3-6), this local decrease in hydroxyl concentration translates into a slower hydrogenation reaction. This reasoning also explains why higher $\mathrm{NaOH}$ concentrations were required to significantly speed up the transfer hydrogenations catalyzed with TPRC (Table 2, entries 3 and 4) compared to hydrogenations catalyzed with TPR (Table 1, entries 4 and 5). Although a significant increase in hydrogenation rate is realizable upon the addition of more base, this strategy is limited by the colloidal stability of the carrier particles. Upon addition of $\mathrm{NaOH}$, the ionic strength of the reaction medium increases, resulting in more efficient screening of surface charges present on the colloids. Consequently, the electrostatic repulsion between individual particles diminishes, eventually leading to the formation of colloidal aggregates. ${ }^{[17]}$ Performing catalytic reactions in aggregated systems is not desirable, as a fraction of the immobilized catalysts will be trapped in the interior of the clusters, thereby lowering the effective catalyst concentration. With the strong dependence of the catalytic performance on the base concentration in mind, a possible strategy we suggest to overcome the requirement of excessive $\mathrm{NaOH}$ concentrations might be to employ colloids with a positively charged surface, instead of the negatively charged particles used throughout this work. This might result in higher hydroxide concentrations near the surface-immobilized catalytic complexes and therefore a significant transfer hydrogenation rate enhancement.

Besides the electrostatic repulsion of the hydroxyl ions close to the colloidal surface, other factors commonly proposed to account for a drop in catalytic activity upon catalyst immobili- zation also apply in the presented example. Although the colloids as a whole are subjected to Brownian motion and therefore mobile, the diffusion of the immobilized catalysts will be significantly slower compared with their molecularly dissolved counterparts. ${ }^{[8, \mathrm{~b}, \mathrm{~d}, \mathrm{f}, \mathrm{g}]}$ This slower diffusion results in less efficient transport of reactants to and/or products from the catalytic centers.

In essence, the colloid can also be regarded as an extremely bulky ligand providing steric hindrance for incoming or eliminated species, thereby slowing down the catalytic conversion. ${ }^{[8 \mathrm{~b}, \mathrm{~d}, \mathrm{f}, \mathrm{g}]}$

Finally, the exact number of catalytic species present in the reaction mixtures conducted with TRPC is not known. The catalyst loading was calculated from an estimated value of the terpyridine loading per particle. Apart from the fact that the measured terpyridine loading might be slightly overestimated (see Scheme 2), the resulting catalyst loading might be even more overvalued, as it is reasonable to assume that not all immobilized terpyridine ligands are converted into the complete, active catalyst. This overestimation of the number of catalysts per particle results in a larger apparent loss of catalytic activity after immobilization, as the activities are compared with hydrogenations performed with higher TPR concentrations. More precise catalyst loadings per particle could possibly be determined by using inductively coupled plasma mass spectrometry (ICP-MS) to improve the comparison between catalytic activity of TPR and TPRC.

Nevertheless, from the results presented in the last two sections, we can conclude that the immobilized form of TPR is catalytically active in the transfer hydrogenation of acetophenone. Although immobilization caused a drop in catalytic activity compared with the homogeneous complex, the decrease is not dramatic and full acetophenone conversion could be obtained after careful optimization of the base concentration. The measured catalytic activities are reasonable from a practical point of view. However, we have to note that more conventional immobilization strategies of transfer hydrogenation catalysts on solid supports, dendrimers, or imprinted polymers generated higher catalytic activities. ${ }^{[18]}$ These higher activities were obtained by using inherently more active (Noyori-type) catalysts and/or significantly higher catalyst loadings.

\section{Immobilization of TPR by adsorption on colloidal surfaces}

Although covalent binding of catalysts to a carrier undoubtedly yields the most robust immobilized systems, one could argue that covalent bonds are not an absolute necessity. In this particular case, the hydrophobic surface of the polystyrene colloids could be used to simply adsorb the catalyst. If the binding energy is sufficiently high, immobilization will be achieved without any particle modification reactions, making this procedure synthetically far less demanding. To probe the feasibility of the adsorption approach, TPR was added to a dispersion of non-functionalized polystyrene seeds (CPs, see the Experimental Section) dispersed in 2-propanol. Complete mixing yielded a homogenous pink dispersion (Figure 5). The number of TPR complexes in this dispersion was comparable to the 


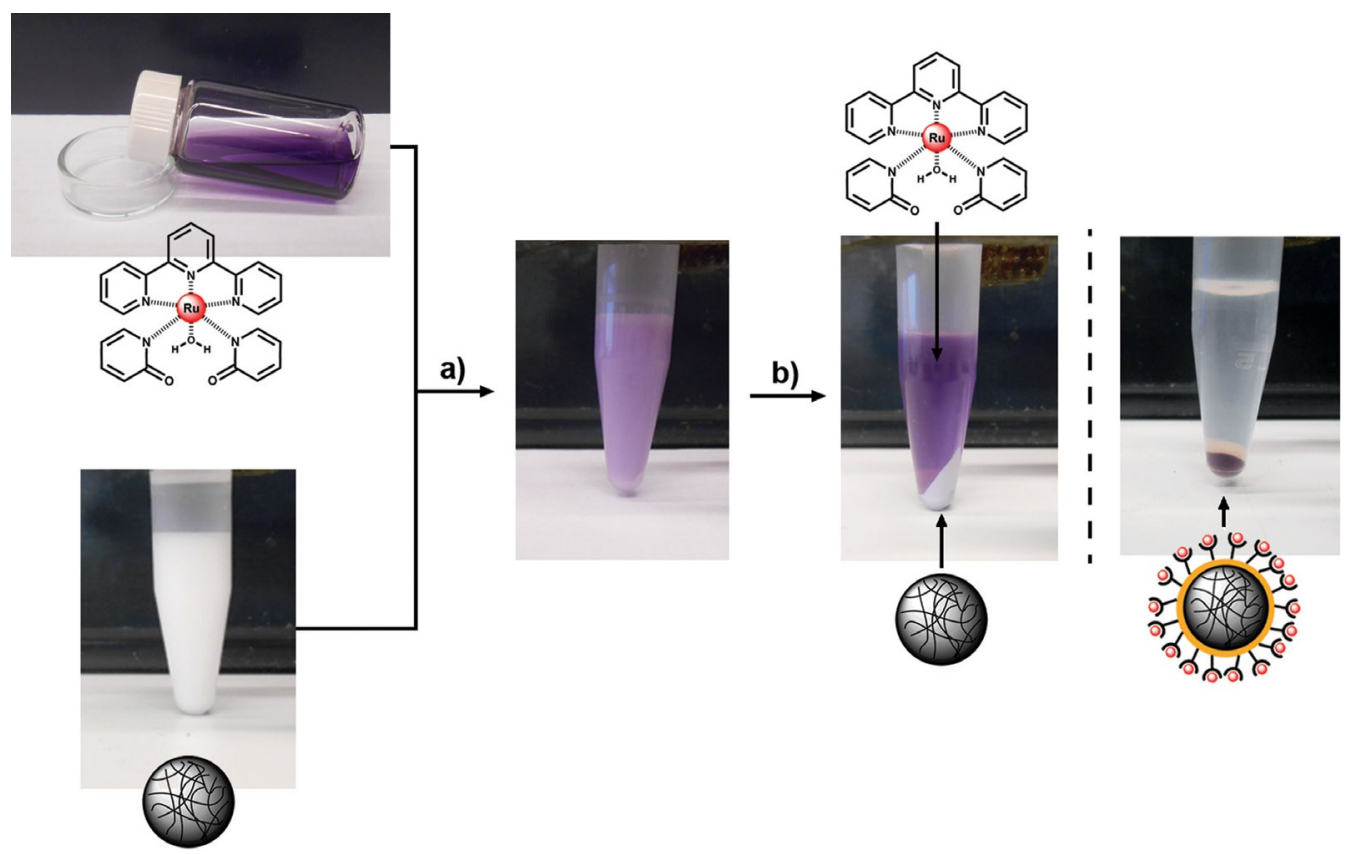

Figure 5. Attempt to immobilize trans-[(terpy)Ru( $\left.\left(\mathrm{NC}_{5} \mathrm{H}_{4} \mathrm{O}-\mathrm{kN}\right)_{2}\left(\mathrm{OH}_{2}\right)\right]$ (TPR) on polystyrene colloids by simple adsorption. Step a): upon mixing a solution of TPR in 2-propanol (left top corner) and non-functionalized polystyrene particles (CPs, left bottom corner), a homogeneous purple/pink dispersion is obtained. Step b): centrifugation of the CPs-TPR mixture yields a purple, TPR-containing supernatant and a white sediment consisting of the polystyrene colloids. The lack of purple color in the sediment strongly indicates the absence of TPR on the particles. If a dispersion containing colloids with TPR covalently bound to their surface (TPRC) was centrifuged, a strongly colored sediment was obtained (picture on right).

number of catalytic entities present in a TPRC dispersion of equal volume and solid content. The pink color was evidently caused by the intense purple color of TPR (Figure 5, left top corner). The resulting dispersion was stirred for $4 \mathrm{~h}$ to facilitate TPR adsorption onto the polystyrene particles. Subsequent centrifugation to separate the particles from the continuous phase resulted in a white sediment and a purple supernatant. This visual observation already strongly suggests that TPR did not adsorb (significantly) onto the colloidal surface, as this would result in a purple/pink sediment. Furthermore, the nonfunctionalized particles showed poor colloidal stability in 2propanol, hampering their use as efficient catalyst carriers even further. These observations are in sharp contrast if compared with a TPRC dispersion. Centrifugation yields a strongly colored sediment in combination with a colorless, transparent supernatant (Figure 5, right panel) showing that the immobilization strategy based on covalent attachment of the catalyst (Scheme 1) is indeed necessary to achieve successful immobilization onto the colloidal particles. On top of actual catalyst binding, TPRC showed good colloidal stability under the reaction conditions required for the transfer hydrogenations. This ensures good dispersion of the carrier particles and therefore maximum accessibility and mobility of the immobilized catalytic species throughout the hydrogenations.

\section{Recycling of catalytic colloids}

As mentioned in the Introduction, the ability to recover the catalyst from the reaction medium is the main advantage of immobilized catalysts in general. To investigate if our system is indeed recyclable, we took the crude reaction mixture of the transfer hydrogenation reaction labelled entry 4 in Table 2 . The particles were easily separated from the crude reaction mixture by centrifugation and redispersed in pure 2-propanol. A second, identical transfer hydrogenation reaction was then performed with these particles (Table 2 , entry 5 ). For both reactions, the conversion was measured after $24 \mathrm{~h}$. The recycled catalyst reached a conversion of $83 \%$, whereas the same reaction with fresh particles yielded $100 \%$ conversion.

This preliminary recycling experiment showed that recovery and reuse of these catalytic particles seems feasible. Most probably a large part of the (apparent) activity loss is caused by either oxygen-mediated catalyst deactivation (see Table 1, entry 2; TPR does not function properly in the presence of oxygen), leaching of ruthenium species or simple loss of catalytic material as a result of centrifugation. The reported conversion after the first recycle step sets, therefore, a lower limit for the catalytic activity of the recycled TPRC. Evidently, more detailed studies to optimize the recycle protocol are required to take full advantage of our immobilization strategy and exploit the proof of principle provided here. Given the focus on the immobilization procedure in the paper, detailed recycling studies are outside the scope of the current manuscript and will be investigated separately.

\section{Conclusions}

We have reported a new strategy for the immobilization of a ruthenium-based transfer hydrogenation catalyst. Stable colloidal polystyrene particles were functionalized with covalently 
attached terpyridine ligands and these particles were subsequently exploited as colloidal ligands to support a rutheniumbased transfer hydrogenation catalyst. The catalyst loading per particle was conveniently measured by determining the terpyridine density by $\mathrm{Fe}^{2+}$ titration. Knowledge of the number of immobilized complexes per particle allowed us to conduct analogous transfer hydrogenations with an equal concentration of the homogeneous catalyst to directly probe the influence of the immobilization procedure on the catalytic activity. Although the catalytic activity decreased after immobilization, significant transfer hydrogenation rates were measured for the reduction of acetophenone to 1-phenylalcohol. The drop in catalytic activity is compensated by preliminary experiments suggesting that our catalytic colloids can be recycled after easy catalyst recovery by centrifugation. Combining the presented results with future work on detailed recycling studies will reveal the true potential of the presented catalytic system.

This novel immobilization strategy shows the potential use of stable colloidal particles as a support for homogeneous catalysts. The colloidal dimensions of the carrier combine high surface area available for catalyst loading, catalyst mobility, and easy separation from the reaction mixture in one immobilization platform.

\section{Experimental Section}

\section{Materials}

Styrene (St, 99\%), divinylbenzene (DVB, 55\% mixture of isomers, tech. grade), 4-vinylbenzyl chloride (VBC, $\geq 90 \%$, tech. grade), sodium bisulfite ( $\mathrm{NaHSO}_{3}$, ACS reagent), 4'-chloro-2, $2^{\prime}: 6^{\prime}, 2^{\prime \prime}$-terpyridine (99\%), 2,2':6',2"-terpyridine (98\%), ruthenium(III) chloride hydrate $\left(\mathrm{RuCl}_{3} \cdot \mathrm{H}_{2} \mathrm{O}, 40.00-49.00 \%\right.$ ruthenium), 2-hydroxypyridine (97\%), dimethylformamide (DMF, >99\%), acetophenone (98\%), 1,10-phenanthroline ( $>99 \%)$, hydroxylamine hydrochloride (puriss p.a.), iron(II) chloride tetrahydrate $\left(\mathrm{FeCl}_{2} \cdot 4 \mathrm{H}_{2} \mathrm{O}\right.$, puriss p.a. $\left.>99 \%\right)$, and $\left[\mathrm{D}_{6}\right]$ acetone $(99.9$ atom $\% \mathrm{D})$ were purchased from Sigma-Aldrich. Powdered potassium hydroxide $(\mathrm{KOH})$, sodium hydroxide $(\mathrm{NaOH})$ pellets, and absolute ethanol $(\mathrm{EtOH})$ were purchased from Merck. 2-Propanol was obtained from Carl Roth and sodium dodecyl sulfate (SDS) from BDH. Finally, potassium persulfate (KPS, $>99 \%$ for analysis) was purchased from Acros Organics. All chemicals were used as received. The water used throughout all syntheses was purified by using a Milli-Q water purification system.

\section{Synthesis of chlorinated seed particles (CPs-Cl)}

Cross-linked polystyrene (CPs) particles were synthesized by using a standard emulsion polymerization method described in the literature. ${ }^{[11]}$ A $500 \mathrm{~mL}$ round-bottom flask equipped with a magnetic stir bar was placed in an oil bath at $80^{\circ} \mathrm{C}$. Water $(200 \mathrm{~mL})$ was charged into the reactor and allowed to reach the bath temperature. Styrene $(23 \mathrm{~mL}, 0.2 \mathrm{~mol}), \mathrm{DVB}(0.68 \mathrm{~mL}, 5 \mathrm{mmol})$, and SDS $(0.25 \mathrm{~g}, 0.9 \mathrm{mmol})$ dissolved in water $(50 \mathrm{~mL})$ were added. The complete mixture was allowed to heat up to the temperature of the bath. Finally, the addition of KPS $(0.39 \mathrm{~g}, 1.4 \mathrm{mmol}$ dissolved in $37.5 \mathrm{~mL}$ water) initiated the polymerization. The reaction was allowed to continue for $24 \mathrm{~h}$ at $80^{\circ} \mathrm{C}$. The resulting dispersion had a solid content of $7 \%$ (measured gravimetrically). The resulting particles had a radius of $125 \mathrm{~nm}$ with a polydispersity of $3.8 \%$ as de- termined with transmission electron microscopy (TEM). The synthesized particles were used as seeds in the second step, in which chlorine groups were introduced at the colloidal surface. ${ }^{[11]}$ Crude seed dispersion $(\mathrm{CPs}, 25 \mathrm{~mL})$ and water $(10 \mathrm{~mL})$ were transferred into a $50 \mathrm{~mL}$ round-bottom flask equipped with a magnetic stir bar. The mixture was degassed with nitrogen for $30 \mathrm{~min}$. VBC ( $1 \mathrm{~mL}, 6.6 \mathrm{mmol}$ premixed with DVB, $20 \mu \mathrm{L}, 0.15 \mathrm{mmol}$ ) was injected under inert atmosphere. The seeds were swollen for $1 \mathrm{~h}$ at $30^{\circ} \mathrm{C}$, after which the temperature was raised to $60^{\circ} \mathrm{C}$. When this temperature was reached, KPS $(0.04 \mathrm{~g}, 0.15 \mathrm{mmol})$ and sodium bisulfite $(0.03 \mathrm{~g}, 0.29 \mathrm{mmol})$ dissolved in water $(2.5 \mathrm{~mL})$ were added to initiate polymerization. The presence of sodium bisulfite shifts the mechanism for radical formation from a purely thermal process to a redox process in which the bisulfite ion acts as a reductor. ${ }^{[10]}$ This way, the polymerization could be performed at a lower temperature, suppressing the undesired hydrolysis of the relatively labile chlorine functionality of 4-vinylbenzyl chloride. The reaction was allowed to run for $4 \mathrm{~h}$. The particles were washed twice by centrifugation (3-4 $\mathrm{h}$ per cycle, $3000 \mathrm{~g}$ ) and redispersed in water. The solid content of the resulting dispersion was adjusted to $5 \%$. A particle radius of $150 \mathrm{~nm}$ and polydispersity of $4.4 \%$ were measured by using TEM. The presence of the chlorine groups was confirmed by using Fourier transform infrared spectroscopy (FTIR; $\tilde{v}=$ $\left.1266 \mathrm{~cm}^{-1}(\mathrm{~s})\right)$ and X-ray photoelectron spectroscopy (XPS): $E_{\mathrm{b}}=$ $200(\mathrm{Cl}), 270 \mathrm{eV}(\mathrm{Cl})$.

\section{Synthesis of terpyridine-functionalized particles (CPs-Terpy)}

A dispersion containing chlorinated polystyrene particles (CPs-Cl) in DMF $(10 \mathrm{~mL}$, solid content $=1 \%)$ was injected into a reaction flask containing powdered $\mathrm{KOH}(20 \mathrm{mg}, 0.35 \mathrm{mmol})$. Sonication was used to aid solubilization of the $\mathrm{KOH}$. The mixture was heated to $60^{\circ} \mathrm{C}$ for $48 \mathrm{~h}$. After hydrolysis, the colloids were washed with DMF (three times) and water (three times) by centrifugation (1-2 $\mathrm{h}$ for each step, $3000 \mathrm{~g}$ ) and redispersion cycles. The chemical composition was monitored with FTIR: $\tilde{v}=3400$ (br) appears, $1266 \mathrm{~cm}^{-1}$ (s) disappears. Attachment of $4^{\prime}$-chloro-2, $2^{\prime}: 6^{\prime}, 2^{\prime \prime}$-terpyridine to the obtained hydrolyzed polystyrene colloids was performed as described in refs. [13] and [19]. The particles containing surface hydroxyl moieties were redispersed in DMF $(10 \mathrm{~mL}$, solid content $=1 \%)$ and subsequently mixed with $\mathrm{KOH}(25 \mathrm{mg}$, $0.44 \mathrm{mmol}$ ) and $4^{\prime}$-chloro-2,2':6',2'-terpyridine (60 mg, $\left.225 \mu \mathrm{mol}\right)$. The resulting reaction mixture was heated to $70^{\circ} \mathrm{C}$ for $48 \mathrm{~h}$. A green/yellow mixture was obtained. These colloids were washed with DMF (three times) and water (three times) by centrifugation (1-2 $\mathrm{h}$ for each step, $3000 \mathrm{~g}$ ) and redispersion cycles. TEM analysis revealed the presence of slightly rough particles with an average radius of $143 \mathrm{~nm}$ and a polydispersity of $4.6 \%$. The presence of terpyridine ligands on the particle surface was verified by addition of $\mathrm{FeCl}_{2}$ to an aqueous dispersion of the obtained particles. After addition, a bright pink dispersion was obtained, which indicated the formation of a coordination complex between $\mathrm{Fe}^{2+}$ and the terpyridine moieties (see Figure 3 a). ${ }^{[13]}$ Centrifugation of the pink dispersion yielded a colorless supernatant and an intensely pink sediment, confirming that the iron-terpyridine complex was indeed immobilized onto the colloids. The presence of iron ions was further confirmed by using energy dispersive X-ray (EDX) spectroscopy on the iron-loaded terpyridine-functionalized colloids (6.4 and $7.1 \mathrm{keV}$ ). Finally, the chemical composition of these particles was probed with FTIR and XPS. FTIR: $\tilde{v}=1600$ (s), 1581 (s), 1561 (s), $1493(\mathrm{~s}), 752(\mathrm{~m}), 697 \mathrm{~cm}^{-1}$ (s). XPS: $E_{\mathrm{b}}=543(\mathrm{O}), 398(\mathrm{~N}), 284 \mathrm{eV}$ (C). 


\section{Determination of terpyridine surface density of CPs-Terpy}

The surface coverage of terpyridines on CPs-Terpy was estimated by performing the following titration experiment. An aqueous solution of $\mathrm{FeCl}_{2} \cdot 4 \mathrm{H}_{2} \mathrm{O}(14 \mu \mathrm{M})$ was added in steps of $2 \mathrm{~mL}$ to an aqueous CPs-Terpy dispersion ( $2 \mathrm{~mL}$, solid content $=1 \%$ ). After each step, the dispersion was sonicated for $5 \mathrm{~min}$ to ensure complete complex formation between the added $\mathrm{Fe}^{2+}$ ions and the immobilized terpyridines. After sonication, the dispersion was centrifuged and $2 \mathrm{~mL}$ of the supernatant was collected and used to determine the concentration of free $\mathrm{Fe}^{2+}$. To measure the concentration of free iron ions, first a UV/Vis spectrum of the supernatant was recorded, which was used as a blank. After recording of the blank spectrum, a 1,10-phenanthroline stock solution $(0.1 \mathrm{~mL}, 5.5 \mathrm{~mm})$ and hydroxylamine. $\mathrm{HCl}$ solution $(20 \mu \mathrm{L}, 1.4 \mathrm{M})$ were added to the supernatant. 1,10-Phenanthroline forms complexes with the $\mathrm{Fe}^{2+}$ ions, which are highly UV active, enabling the detection of $\mathrm{Fe}^{2+}$ concentrations down to ppm levels. ${ }^{[14]}$ The presence of hydroxylamine ensures that all iron ions are present in oxidation state +2 . The resulting solution was measured again by using UV/Vis spectroscopy. After subtraction of the blank spectrum, the absorbance at $508 \mathrm{~nm}$ was indicative for the $\mathrm{Fe}^{2+}$ concentration. The relationship between iron concentration and absorbance was previously determined by measuring a concentration series of the $\mathrm{Fe}^{2+}-1,10-$ phenanthroline complex (see the Supporting Information). If UV/ Vis spectroscopy did not show any $\mathrm{Fe}^{2+}$ ions, a new shot of the $\mathrm{FeCl}_{2}$ stock solution was added to the same CPs-Terpy colloids. This procedure was repeated until free $\mathrm{Fe}^{2+}$ ions were measured. Based on the total amount of ions added to the particles minus the free ions, which were measured in the last titration step, the total iron uptake per particle could be calculated. In this calculation, we assumed that the particles were perfectly spherical with a radius of $143 \mathrm{~nm}$ and had the density of pure polystyrene $(\rho=$ 1.05). Furthermore, we assumed formation of monodentate ironterpyridine complexes exclusively. A resulting terpyridine surface density of one terpyridine moiety per $4 \mathrm{~nm}^{2}$ was obtained.

\section{Synthesis of homogeneous catalyst (trans-[(terpy)Ru(N- $\left.\left.\mathrm{C}_{5} \mathrm{H}_{4} \mathrm{O}-\kappa \mathrm{N}\right)_{2}\left(\mathrm{OH}_{2}\right)\right](\mathrm{TPR})$ )}

First, $\left[\mathrm{RuCl}_{3}\right.$ (terpy)] was obtained by complexation of $\mathrm{Ru}_{3} \mathrm{Cl}$ with $2,2^{\prime}: 6^{\prime}, 2^{\prime \prime}$-terpyridine as described by Sullivan et al. ${ }^{[20]}$ The complex was isolated as a dark-brown solid. Yield: $68.8 \%$; FTIR: $\tilde{v}=1595$ (s), $1564(\mathrm{~m}), 1537(\mathrm{~m}), 1444$ (s), $774(\mathrm{~s}), 729 \mathrm{~cm}^{-1}$ (s). The formed $\left[\mathrm{RuCl}_{3}\right.$ (terpy)] was used for the preparation of the molecular transfer hydrogenation catalyst by following a method reported by Kelson et al. ${ }^{[10]}$ The catalyst was isolated as dark-purple crystals. Yield: 93\%; FTIR: $\tilde{v}=1604$ (s), 1539 (s), 1470 (s), 1443 (s), 1375 (s), $1274(\mathrm{~s}), 1009(\mathrm{~s}), 965(\mathrm{w}), 845(\mathrm{~m}), 762(\mathrm{~m}), 738 \mathrm{~cm}^{-1}(\mathrm{~m}) ;{ }^{1} \mathrm{H}$ NMR $\left(400 \mathrm{MHz},\left[\mathrm{D}_{6}\right.\right.$ ]acetone, $\left.25^{\circ} \mathrm{C}\right): \delta=9.24(2 \mathrm{H}, \mathrm{m}), 8.48(4 \mathrm{H}, \mathrm{m}), 7.93$ $(2 \mathrm{H}, \mathrm{td}, J=7.5$ and $1.6 \mathrm{~Hz}), 7.76(1 \mathrm{H}, \mathrm{t}, J=8 \mathrm{~Hz}) .7 .60(2 \mathrm{H}, \mathrm{m}), 6.77$ $(2 \mathrm{H}, \mathrm{m}),, 5.77(2 \mathrm{H}, \mathrm{m}), 5.75(2 \mathrm{H}, \mathrm{m}), 5.45 \mathrm{ppm}(2 \mathrm{H}, \mathrm{m})$.

\section{Synthesis of immobilized catalyst (TPRC)}

The synthetic procedure towards the immobilized catalyst was analogous to the one described for preparation of the homogeneous catalyst (TPR). The only key difference was the use of terpyridine ligands, which were covalently bound to polystyrene colloids (CPs-Terpy) instead of free molecular terpyridines. An excess of $\mathrm{RuCl}_{3} \cdot \mathrm{xH}_{2} \mathrm{O}(20 \mathrm{mg}, 79-97 \mu \mathrm{mol})$ was added to a dispersion containing terpyridine-functionalized colloids (CPs-Terpy, $10 \mathrm{~mL}$, solid content $1 \%=0.094 \mu \mathrm{mol}$ terpyridine $\mathrm{mL}^{-1}$ ) in ethanol. The disper- sion was heated at reflux for $3 \mathrm{~h}$, after which the particles were washed with water (two times) and ethanol (two times) by centrifugation $(0.5-2 \mathrm{~h}$ for each step, $3000 \mathrm{~g})$ and redispersion cycles. The colloids were analyzed with FTIR and XPS. FTIR: $\tilde{v}=1600$ (s), $1493(\mathrm{~s}), 752(\mathrm{~m}), 697 \mathrm{~cm}^{-1}(\mathrm{~s})$. XPS: $E_{\mathrm{b}}=543(\mathrm{O}), 461(\mathrm{Ru}), 398(\mathrm{~N})$, 284 (C), $270(\mathrm{Cl}), 200 \mathrm{eV}(\mathrm{Cl})$. The particles containing ruthenium coordinated terpyridine ligands were redispersed in ethanol $(10 \mathrm{~mL})$ and 2-hydroxypyridine $(0.42 \mathrm{mg}, 4.4 \mu \mathrm{mol})$ was added. The dispersion was heated at reflux for $1 \mathrm{~h}$, after which an aqueous $\mathrm{NaOH}$ solution $(5 \mathrm{~mL}, 300 \mathrm{~mm}$ ) was injected into the reaction mixture. Reflux was continued for two additional hours. After refluxing, the colloids were washed once with water, ethanol, and 2-propanol by centrifugation $(0.5-2 \mathrm{~h}$ for each cycle, $3000 \mathrm{~g})$ and redispersion cycles. TEM analysis revealed the presence of rough particles with an average radius of $142 \mathrm{~nm}$ and a polydispersity of $5 \%$. The chemical composition was probed with FTIR and XPS. FTIR: $\tilde{v}=$ 1607 (s), 1550 (br), $1382 \mathrm{~cm}^{-1}$ (br). XPS: $E_{\mathrm{b}}=543(\mathrm{O}), 461(\mathrm{Ru}), 398$ (N), $284 \mathrm{eV}(\mathrm{C})$.

\section{General procedure for transfer hydrogenation reactions catalyzed with TPR(C)}

TPR dissolved in 2-propanol (1 mL, catalyst concentration was varied: see Table 1) or a 2-propanol TPRC dispersion $(1 \mathrm{~mL}$, solid content $=2 \%, \approx 0.188 \mu \mathrm{mol}$ catalytic moieties) was introduced into a Schlenk flask and degassed by careful evacuation and refilling with nitrogen. A degassed $\mathrm{NaOH}$ solution in 2-propanol $(2 \mathrm{~mL})$ was injected. $\mathrm{NaOH}$ concentrations were varied for both reactions conducted with TPR (Table 1) and TPRC (Table 2). The resulting mixture was heated at reflux for 30 min under a nitrogen atmosphere. A color change from purple to yellow was observed. Finally, a degassed acetophenone stock solution in 2-propanol $(2 \mathrm{~mL}, 20 \mathrm{~mm})$ was added. Conversions of acetophenone were measured with gas chromatography-mass spectrometry (GC-MS). Typically, samples were withdrawn from the mixture 2 and $24 \mathrm{~h}$ after acetophenone injection. If necessary, the samples were diluted with 2-propanol before conversion determination. For the transfer hydrogenation performed with TPRC, GC-MS measurements were conducted on the supernatants obtained after centrifugation $(5 \mathrm{~min}, 11000 \mathrm{~g})$ of the samples. Only the top part of the supernatants was used for GC-MS measurements to ensure the complete absence of colloids.

\section{Recycling of TPRC}

TPRC colloids that were previously used in a catalytic reaction were separated from the reaction mixture by centrifugation $(5 \mathrm{~min}$, $11000 \mathrm{~g}$ ). The colloids were then redispersed in 2-propanol and a new catalytic reaction was performed as described in the previous subsection. The conversion of acetophenone after $24 \mathrm{~h}$ of reaction was measured with GC-MS and compared with the conversion obtained after the first catalytic reaction performed with the same TPRC colloids.

\section{Measuring adsorption of TPR on polystyrene colloids}

Non-functionalized colloids (CPs, $1 \mathrm{~mL}$, solid content $=1 \%)$ were centrifuged (10 min, $11000 \mathrm{~g}$ ). The supernatant was removed and the particles were subsequently redispersed in a solution of TPR in 2-propanol ( $1 \mathrm{~mL}, 0.188 \mathrm{~mm}$ ). The resulting dispersion was stirred for $4 \mathrm{~h}$ to provide sufficient time for potential TPR adsorption. After this period, the dispersion was centrifuged (10 min, $11000 \mathrm{~g}$ ) and the color change of the sediment was monitored by eye. Ad- 
sorption of the purple complex onto the white polystyrene particles should result in a similar purple sediment as observed for TPRC (see Figure 5). However, the sediment was not colored, strongly suggesting complete lack of catalyst adsorption onto the surface of the non-functionalized polystyrene particles.

\section{Characterization}

Transmission electron microscopy (TEM) pictures were recorded with a Philips Technai10 electron microscope typically operating at $100 \mathrm{kV}$. Bright field images were recorded by using a SIS Megaview IICCD camera. The samples were prepared by drying a drop of diluted, aqueous particle dispersion on top of polymer-coated copper grids. Energy dispersive X-ray (EDX) spectra were recorded with a TechnaiF20 from FEl with an acceleration voltage of $200 \mathrm{kV}$. The microscope was equipped with an EDX system from EDAX and used Emispec software in STEM mode. X-ray photoelectron spectroscopy (XPS) was performed by using a Thermo-Scientific KAlpha, equipped with a monochromatic small-spot X-ray source and a $180^{\circ}$ double focusing hemispherical analyzer with a 128channel detector. Spectra were obtained by using an aluminum anode $\left(\mathrm{AlK}_{\alpha}=1486.6 \mathrm{eV}\right)$ operating at $72 \mathrm{~W}$ and a spot size of $400 \mu \mathrm{m}$. Survey scans were measured at a constant pass energy of $200 \mathrm{eV}$ and region scans at $50 \mathrm{eV}$. The background pressure was $2 \times 10^{-9} \mathrm{mbar}$ and during measurement $3 \times 10^{-7} \mathrm{mbar}$ argon was used because of the charge compensation dual beam source. Infrared (IR) spectra were recorded by using a PerkinElmer FTIR/FIR Frontier Spectrometer. The attenuated total reflectance (ATR) mode was used. Measurements were performed on powders obtained by drying the particle dispersion. UV/Vis spectra were measured with a PerkinElmer Lambda-35 spectrophotometer using $1 \mathrm{~cm}$ quartz cuvettes. ${ }^{1} \mathrm{H}$ NMR spectra were recorded by using a Varian MRF400 $400 \mathrm{MHz}$ NMR machine. Samples were dissolved in $\left[D_{6}\right]$ acetone. Gas chromatography-mass spectrometry (GC-MS) measurements were performed with a PerkinElmer Austosystem XL Gas Chromatograph equipped with a PE-17 column (50\% phenyl, 50\% methylpolysiloxane; $30 \mathrm{~m} \times 0.23 \times 0.5 \mu \mathrm{m})$. Samples withdrawn from the transfer hydrogenation reactions were stored in the freezer $\left(-8^{\circ} \mathrm{C}\right)$. In all measured traces, only signals corresponding to acetophenone and the product (1-phenylethanol) were observed, indicating the absence of side products and high selectivity of the (immobilized) catalyst. The obtained conversions were therefore indicative for the yield.

\section{Acknowledgments}

This work was financially supported by The Netherlands Organization for Scientific Research (NWO). The authors thank Judith van Wijk (Eindhoven University of Technology) for the XPS analysis and Hans Meeldijk (Utrecht University) for performing the EDX and STEM measurements.

Keywords: catalyst immobilization - colloids · supported catalysis - transfer hydrogenation
[1] R. J. Hunter in Foundations of Colloid Science, 2nd ed., Oxford University Press, New York, 2009.

[2] L. González-Urbina, K. Baert, B. Kolaric, J. Pérez-Moreno, K. Clays, Chem Rev. 2012, 112, 2268-2285.

[3] M. Douaire, M. I. T. Norton, J. Sci. Food Agric. 2013, 93, 3147-3154.

[4] P. H. H. Hermkens, H. C. J. Ottenheijm, D. Rees, Tetrahedron 1996, 52 $4527-4554$.

[5] H. Kawaguchi, Prog. Polym. Sci. 2000, 25, 1171-1210.

[6] L. Hu, Z. Mao, C. Gao, J. Mater. Chem. 2009, 19, 3108-3115.

[7] E. M. Claesson, N. C. Mehendale, R. J. M. Klein Gebbink, G. van Koten A. P. Philipse, J. Magn. Magn. Mater. 2007, 311, 41-45.

[8] a) I. Chorkendorff, J. W. Niemantsverdriet in Concepts of Modern Catalysis and Kinetics, 1st ed., Wiley-VCH, Weinheim, 2003; b) P. Barbaro, F. Liguori in Heterogenized Homogeneous Catalysts for Fine Chemicals Production, Catalysis by Metal Complexes, Vol. 33, Springer, Dordrecht, 2010; c) R. Šebesta in Enantioselective Homogeneous Supported Catalysis, RSC Green Chemistry series, No. 15, The Royal Society of Chemistry, Cambridge, 2012; d) A. Choplin, F. Quignard, Coord. Chem. Rev. 1998, 178 180,1679-1702; e) J. M. Brown, H. Molinari, Tetrahedron Lett. 1979, 20, 2933-2936; f) F. R. Hartley, P. N. Vezey, Adv. Organomet. Chem. 1977, 15, 189-234; g) X. S. Zhao, X. Y. Bao, W. Guo, F. Y. Lee, Mater. Today 2006, 9, $32-39$.

[9] a) S. Gladiali, E. Alberico, Chem. Soc. Rev. 2006, 35, 226-236; b) R Noyori, S. Hashiguchi, Acc. Chem. Res. 1997, 30, 97-102; c) R. A. W. Johnstone, A. H. Wilby, Chem. Rev. 1985, 85, 129-170; d) T. Naota, H. Takaya, S.-I. Murahashi, Chem. Rev. 1998, 98, 2599-2660; e) G. Brieger, T. J. Nestrick, Chem. Rev. 1974, 74, 567-580; f) O. Pàmies, J.-E. Bäckvall, Chem. Eur. J. 2001, 7, 5052-5058.

[10] E. P. Kelson, P. P. Phengsy, J. Chem. Soc. Dalton Trans. 2000, 4023-4024.

[11] B. G. P. van Ravensteijn, M. Kamp, A. van Blaaderen, W. K. Kegel, Chem. Mater. 2013, 25, 4348-4353.

[12] J. R. Ebdon, T. N. Huckerby, T. C. Hunter, Polymer 1994, 35, 4659-4664.

[13] U. S. Schubert, A. Alexeev, P. R. Andres, Macromol. Mater. Eng. 2003, 288, $852-860$.

[14] W. B. Fortune, M. G. Mellon, Ind. Eng. Chem. Anal. Ed. 1938, 10, 60-64.

[15] J. S. M. Samec, J.-E. Bäckvall, P. G. Andersson, P. Brandt, Chem. Soc. Rev. 2006, 35, 237-248.

[16] W. Baratta, K. Siwga, P. Rigo, Chem. Eur. J. 2007, 13, 7479-7486.

[17] a) E. J. W. Verwey, J. T. G. Overbeek in Theory of the Stability of Lyophobic Colloids, 2nd ed., Dover Publications, Mineola, NY, 1999; b) J. N. Israelachvili in Intermolecular and Surface Forces, 3rd ed., Academic Press, Amsterdam, 2011.

[18] a) P. N. Liu, P. M. Gu, F. Wang, Y. Q. Tu, Org. Lett. 2004, 6, 169-172; b) C. Saluzzo, M. Lemaire, Adv. Synth. Catal. 2002, 344, 915-928; c) X. Li, X Wu, W. Chen, F. E. Hancock, F. King, J. Xiao, Org. Lett. 2004, 6, 3321 3324; d) K. Polborn, K. Severin, Chem. Eur. J. 2000, 6, 4604-4611; e) Y.-C. Chen, T.-F. Wu, L. Jiang, J.-G. Deng, H. Liu, J. Zhu, Y.-Z. Jiang, J. Org. Chem. 2005, 70, 1006-1010; f) Q. Sun, X. Meng, X. Liu, X. Zhang, Y. Yang, Q. Yang, F.-S. Xiao, Chem. Commun. 2012, 48, 10505-10507; g) X. Huang, J. Y. Ying, Chem. Commun. 2007, 1825-1827; h) Y. Arakawa, A. Chiba, N. Haraguchi, S. Itsuno, Adv. Synth. Catal. 2008, 350, 2295-2304; i) N. Haraguchi, K. Tsuru, Y. Arakawa, S. Itsuno, Org. Biomol. Chem. 2009, $7,69-75$.

[19] a) D.-W. Yoo, S.-K. Yoo, C. Kim, J.-K. Lee, J. Chem. Soc. Dalton Trans. 2002, 3931 -3932; b) U. Sampath, W. C. Putnam, T. A. Osiek, S. Touami, J. Xie, D. Cohen, A. Cagnolini, P. Droege, D. Klug, C. L. Barnes, A. Modak, J. K. Bashkin, S. S. Jurisson, J. Chem. Soc. Dalton Trans. 1999, 2049-2058.

[20] B. P. Sullivan, J. M. Calvert, T. J. Meyer, Inorg. Chem. 1980, 19, 1404 1407

Manuscript received: September 3, 2016

Revised: October 10, 2016

Accepted Article published: October 27, 2016

Final Article published: December 21, 2016 